\title{
Effects of Upper Limb Stabilization Exercise on Pain Control and Improvement of Function in Shoulder Dislocation Patients
}

\author{
Dae-hwan Lee ${ }^{1}$, Jung-ho Lee ${ }^{2}$ and Youn-bum Sung ${ }^{3 *}$ \\ ${ }^{1}$ Dept. Physical Therapy, Daegu Univ., 201, Daegudae-ro, Jillyang-eup, Gyeongsan- \\ si, Gyeongsangbuk-do, 38453, Republic of Korea \\ ${ }^{2}$ Dept. Physical Therapy, Kyungdong Univ., Bongpo-ri, Toseong-myeon, Goseong- \\ gun, Gangwon-do, 219-705, Republic of Korea \\ ${ }^{3}$ Dept. Physical Therapy, Daegu Univ., 201, Daegudae-ro, Jillyang-eup, Gyeongsan- \\ si, Gyeongsangbuk-do, 38453, Republic of Korea \\ 1'dhlee8510@naver.com, ${ }^{2}$ ljhcivapt@naver.com, ${ }^{3}$ playeryoon@naver.com
}

\begin{abstract}
In this paper, we investigated the effects of stability exercise of the upper extremities after shoulder dislocation patients on pain and function. The control group $(n=15)$ performed traditional physical therapy, and the experimental group $(n=15)$ performed shoulder stabilization exercises for 30 minutes, and the subjects were evaluated using Visual Analog Scale (VAS), Constant-Murley Scale (CMS), and Simple Shoulder Test (SST). In the experimental group, the power items of CMS, SST and Active Abduction were statistically significant compared to the control group. Shoulder stabilization exercises can help pain and functional recovery in shoulder dislocation patients.
\end{abstract}

Keywords: Stabilization exercise, Swiss ball exercise, Upper limb function

\section{Introduction}

The shoulder joint is one of a few joints connected to muscles and tendons in a human body. Although muscles and tendons enable the movement of arms in a wide range, only one-third of the humeral head is in contact with the glenoid fossa in the scapula, which means that the shoulder joint is a joint that is very weak in terms of stability. Because of this, the shoulder joint is the most commonly dislocated joint in the human body, and dislocation may also incur hand injuries [1].

Primarily, the connective tissues, such as the joint capsule, ligament, and glenoid labrum, align and protect the joints for bones. Secondarily, muscle tone and contraction protect the joints, thereby preventing dislocation or subluxation.

Many shoulder joint dislocations are anterior shoulder dislocations. Anterior shoulder dislocations occur due to direct external injuries or during falls on an outstretched hand. The injury type is divided into a Bankart lesion and a Hill-Sachs lesion. A Bankart lesion is a detachment of the glenoid labrum or the articular capsules from the anterior glenoid cavity, which can be seen by arthroscopy or magnetic resonance imaging. A Hill-Sachs lesion is an injury in the articular cartilage or an osteochondral injury, which can occur due to the

Article history:

Received (May 12, 2020), Review Result (June 17, 2020), Accepted (July 20, 2020) 
collision of the posterolateral side of the humeral head with the glenoid fossa. A Hill-Sachs lesion is a bone injury that can be seen by general imaging methods. To treat patients with anterior shoulder dislocation, surgical, non-surgical, and conservative treatments can be applied. Among them, patients who chose surgical treatments had a lower recurrence rate than patients who chose other treatments [2][3].

The soft tissue around the shoulder, such as ligaments, capsules, and the glenoid labrum, can add stability to the shoulder, and the rotator cuff is the typical muscle to play that role. Generally, shoulder dislocation tears or damages the glenoid labrum, surrounding soft tissues, or the long head of the biceps brachii muscle [4]. Thus, surgery must be conducted to fix the soft tissues. As surgical methods, Bankart repair, joint capsule repair, and thermal capsulorrhaphy can be conducted. It is important to recover the limited joint range of motion to a normal range and obtain muscle strength and stability after surgery. At the early days after surgery, it is generally desirable to apply muscle strength exercises after using hyperthermia, cryotherapy, and electrotherapy to control the pain and relax the soft tissues [5].

The stabilization exercise for shoulder joints is an exercise without too much pressure on shoulder joints, which can be relatively safely applied to patients immediately after shoulder joint surgery. The stabilization exercise of shoulder joints is used to correct functional impediments, such as the abnormal location of the scapula and abnormal movements, to provide the overall stability throughout the shoulder girdle [6].

The Swiss Ball is a widely used tool in core stability exercises. Marshall et al. (2005) reported that the stabilization exercise using the Swiss Ball had a significant impact on the activation of the rectus abdominis [7]. They also proved that the activation of the trapezius was higher when the wall-press exercise using the Swiss Ball was conducted [8]. It has also been reported that closed chain exercises are functionally more advantageous for proprioception stimulation than open chain exercises. The stability of the scapulothoracic joint is more important than the glenohumeral joint in the shoulder joints. The scapulothoracic joint is not a true joint, but it plays an essential role in the mobility and stability of the shoulder joint [9].

Thus, the stability in the scapulothoracic joint was attempted first by applying the shoulder joint stabilization exercise for patients with the dislocation of the shoulder joint [10]. The muscular atrophy and shortness in surrounding the shoulder joints, such as the teres minor, teres major, subscapularis, rhomboids, serratus anterior muscle, and lower trapezius, can be prevented and muscle strength can be strengthened through the stabilization exercise [11]. Previous studies have been conducted on the comparison of recurrence rates between nonsurgical and surgical treatments [4]. However, few studies have been conducted on pain control, joint range of motion, and functional assessment of the shoulder joint after the surgery of patients with shoulder dislocation. This study aims to determine the effects of the shoulder joint stabilization exercise on the pain and functions of patients with shoulder dislocation after surgery.

\section{Subjects and methods}

\subsection{Subjects}

This study selected 30 shoulder dislocation patients who were no more than three months after their shoulder dislocation. The subjects were recruited by people living in $\mathrm{C}$ city, with 19 women, and 11 men. The age range of the subjects was set from 55 to 64 years of age. 


\subsection{Research design}

The shoulder is a joint that has a high risk of damage such as dislocation or a dislocation, instead of having high mobility. People with limited shoulder movements have many restrictions on their daily lives, especially those with dislocation patients with weakened surrounding tissues that protect their shoulders. Therefore, it improves the quality of activities of daily life of dislocated patients by stabilizing their shoulders.

This study examined the effects of conventional physical therapy and shoulder stabilization exercise on shoulder pain and range of motion and functional activity in dislocated patients. The subjects matched the ratio of men and women, but randomly assigned groups, and all experiences were the same except for intervention for experimentation with blind test. The application of treatment to the subjects was made by physical therapists with more than three years of clinical experience.

\subsection{Intervention for each group}

The subjects had physiotherapy for $40 \mathrm{~min}$, and the control group $(\mathrm{n}=15)$ had traditional physiotherapy for $30 \mathrm{~min}$. while the experimental group $(\mathrm{n}=15)$ had shoulder joint stabilization exercises for $30 \mathrm{~min}$. three times a week for six weeks. The researchers evaluated the pain, functions, and the joint range of motion of the subjects. The treatment was conducted by two experienced physiotherapists with more than 10 years of clinical experience.

More specifically, both the experimental and control groups had hyperthermia for $20 \mathrm{~min}$. followed by transcutaneous electrical nerve stimulation for $10 \mathrm{~min}$. and laser treatment for 10 min. Then, each group had their group intervention for $30 \mathrm{~min}$. For the intervention in each group, the experimental and control groups conducted shoulder joint stabilization and general exercises, respectively.

\subsection{Pain and function assessment}

To assess the pain level of the subjects before and after the treatment, a $100 \mathrm{~mm}$ Visual Analog Scale (VAS) was used in which patients expressed their pain level in the shoulder joint by seeing the VAS. To assess the functions of the shoulder joint, the Constant-Murley Scale (CMS), which is known as a standardized clinical measurement method, was employed. The assessment items were pain level, joint range of motion, activities of daily living, and muscle strength of the shoulder joint. The total score was 100 points, which were divided into 35 points of subjective elements and 65 points of objective elements. The subjective elements were pain level (15 points), activities of daily living (20 points), and the objective elements were a range of motion (flexion, abduction, external rotation, and internal rotation, whose possible total score was 40 points), and shoulder joint muscle strength ( 25 points). The higher the item or total score was, the better the shoulder functions were. To measure a range of motion, a goniometer was used to measure the active flexion and extension in the shoulder joint.

\subsection{Intervention procedure}

For 15 subjects of the control group, $20 \mathrm{~min}$. of hyperthermia, $15 \mathrm{~min}$. of transcutaneous electrical nerve stimulation, and $10 \mathrm{~min}$. of laser were applied as general physiotherapy treatments to control the pain, and then $20 \mathrm{~min}$. of interferential current therapy, and five min. of ultrasound were applied. For 15 subjects of the experimental group, the same general physiotherapy treatments were applied, followed by a stabilization exercise. The shoulder 
joint stabilization exercise was conducted on a patient who lied flat on their back on the treatment table, and the patient's shoulder joint was located with the Swiss Ball while flexing the knee joints at $90^{\circ}$. Both feet were contacted at the bottom of the treatment table. The Swiss Ball was fixed through adduction and retraction of the shoulder joint by the patients. Patients with low back pain wore abdominal support to relieve the pressure on their waist.

In addition, the patients had their elbow joints flexed at $90^{\circ}$ while standing and fixed the Swiss Ball against the wall in the treatment room using the wrist of their lower arms and leaning forward. Here, the therapist instructed the patients to push their arm outward to maintain the position. All exercises had patients maintain their posture for 10 seconds followed by five seconds of rest; this was done 10 times to complete one set. For the scapula stabilization exercise and the shoulder joint stabilization exercise, three sets were conducted, and three min. of rest were given between sets. For the stabilization of the scapula and shoulder joint, the therapist let the patients apply resistance against various directions. The treatments were conducted three times a week for six weeks, for a total of 18 exercises.

\subsection{Statistical analysis}

For the data processing in this study, descriptive statistics were conducted to determine the general characteristics of the subjects. A paired sample t-test was conducted to determine the difference in functional improvement before and after the treatment of the experimental and control groups. An independent t-test was conducted to determine the treatment effect between the groups.

The data were analyzed using SPSS version 20.0 for Windows, and the significance level $\alpha$ was set to 0.05 .

\section{Results}

There were 30 subjects participating in this study; eight males and 22 females, and the mean age, mean weight, and mean height in the experimental group were $59.73 \pm 6.09$ years, $58.27 \pm 6.46 \mathrm{~kg}$, and $158.33 \pm 7.98 \mathrm{~cm}$, and those of the control groups were $61.20 \pm 4.81$ years, $57.87 \pm 5.84 \mathrm{~kg}$, and $160.40 \pm 5.67 \mathrm{~cm}$. There was no statistically significant difference between them in the homogeneity test $(\mathrm{p}>.05)$ [Table 1].

Table 1. General characters of subjects

\begin{tabular}{|c|c|c|c|}
\hline & Experimental group (n=15) & Control group (n=15) & $p$ \\
\hline Age & $59.73 \pm 6.09$ & $61.20 \pm 4.81$ & .470 \\
\hline Body weight & $58.27 \pm 6.46$ & $57.87 \pm 5.84$ & .860 \\
\hline Body height & $158.33 \pm 7.98$ & $160.40 \pm 5.67$ & .420 \\
\hline Sex (female / male) & $(9 / 6)$ & $(10 / 5)$ & \\
\hline Pain position (left / right) & $(5 / 10)$ & $(3 / 12)$ & \\
\hline
\end{tabular}

The results of the experimental group before and after the treatment exhibited that the joint range of motion in active flexion, active abduction, and the VAS results were statistically and significantly different, and all items in the CMS were statistically and significantly different $(\mathrm{p}<.05)[$ Table 2]. 
Table 2. Experimental groups analysis

\begin{tabular}{|c|c|c|c|c|c|}
\hline & & Pre-test $(n=15)$ & Post-test $(n=15)$ & $\mathrm{t}$ & $\mathrm{p}$ \\
\hline \multicolumn{2}{|c|}{ Active Flexion } & $77.33 \pm 11.47$ & $86.67 \pm 13.18$ & -2.068 & $.048 *$ \\
\hline \multicolumn{2}{|c|}{ Active Abduction } & $73.33 \pm 5.56$ & $83.00 \pm 7.51$ & -4.005 & $.000^{*}$ \\
\hline \multicolumn{2}{|c|}{ Visual Analog Scale } & $57.33 \pm 12.80$ & $45.33 \pm 13.56$ & 2.493 & $.019 *$ \\
\hline \multicolumn{2}{|c|}{ Simple Shoulder Test } & $10.80 \pm 1.52$ & $9.53 \pm 2.00$ & 1.955 & .061 \\
\hline \multirow{5}{*}{ CMS } & PAIN & $6.33 \pm 2.97$ & $8.67 \pm 2.97$ & -2.153 & $.040^{*}$ \\
\hline & $\mathrm{ADL}$ & $11.73 \pm 1.83$ & $14.53 \pm 2.56$ & -3.446 & $.002 *$ \\
\hline & ROM & $17.60 \pm 5.19$ & $22.27 \pm 5.44$ & -2.402 & $.023 *$ \\
\hline & POWER & $13.47 \pm 2.80$ & $17.73 \pm 3.03$ & -4.002 & $.000^{*}$ \\
\hline & Total & $49.47 \pm 9.46$ & $64.27 \pm 9.18$ & -4.348 & $.000^{*}$ \\
\hline
\end{tabular}

${ }^{*} p<0.05$, VAS =Visual Analogue Scale, SST =Simple Shoulder Test, ADL =Activities of Daily Living,

CMS = Constant-Murley scale The results of the control group before and after the treatment exhibited that the joint range of motion in active abduction and the VAS results were statistically and significantly different, and only the total score in the CMS was statistically and significantly different $(\mathrm{p}<.05)$ [Table 3].

Table 3. Control groups analysis

\begin{tabular}{|c|c|c|c|c|c|}
\hline & & Pre-test $(n=15)$ & Post-test $(n=15)$ & $\mathrm{t}$ & $\mathrm{p}$ \\
\hline \multicolumn{2}{|c|}{ Active Flexion } & $77.33 \pm 14.38$ & $85.33 \pm 15.41$ & -1.470 & .153 \\
\hline \multicolumn{2}{|c|}{ Active Abduction } & $77.67 \pm 9.04$ & $94.33 \pm 9.98$ & -4.795 & $.000^{*}$ \\
\hline \multicolumn{2}{|c|}{ Visual Analog Scale } & $61.33 \pm 11.87$ & $50.67 \pm 14.13$ & 2.239 & $.033^{*}$ \\
\hline & $10.07 \pm 1.53$ & $10.13 \pm 1.25$ & -.131 & .897 \\
\hline \multirow{5}{*}{ CMS } & PAIN & $7.67 \pm 3.20$ & $9.00 \pm 2.07$ & -1.355 & .186 \\
\hline & ADL & $11.73 \pm 2.12$ & $13.33 \pm 2.35$ & -1.958 & .060 \\
\hline & $\mathrm{ROM}$ & $19.47 \pm 5.37$ & $21.87 \pm 5.21$ & -1.243 & .224 \\
\hline & POWER & $14.80 \pm 3.26$ & $16.40 \pm 3.09$ & -1.381 & .178 \\
\hline & Total & $52.67 \pm 10.05$ & $61.67 \pm 9.97$ & -2.462 & $.020 *$ \\
\hline
\end{tabular}

${ }^{*} p<0.05$, VAS = Visual Analogue Scale, SST =Simple Shoulder Test, ADL =Activities of Daily Living,

CMS = Constant-Murley scale The difference before and after treatment between the experimental and control groups was statistically significant in the joint range of motion in active abduction and simple shoulder test, and only the power item in the CMS was statistically and significantly different $(\mathrm{p}<.05)$ [Table 4].

Table 4. Pre-post difference analysis in each group

\begin{tabular}{|c|c|c|c|c|c|}
\hline & & Experimental group $(\mathrm{n}=15)$ & Control group $(n=15)$ & $\mathrm{t}$ & $\mathrm{p}$ \\
\hline \multicolumn{2}{|c|}{ Active Flexion } & $9.33 \pm 4.95$ & $8.00 \pm 3.16$ & .879 & .387 \\
\hline \multicolumn{2}{|c|}{ Active Abduction } & $9.67 \pm 3.99$ & $16.67 \pm 4.88$ & -4.299 & $.000 *$ \\
\hline \multicolumn{2}{|c|}{ Visual Analog Scale } & $-12.00 \pm 4.14$ & $-10.67 \pm 4.58$ & -.837 & .410 \\
\hline \multicolumn{2}{|c|}{ Simple Shoulder Test } & $-1.27 \pm 1.16$ & $0.07 \pm 1.44$ & -2.793 & $.009 *$ \\
\hline \multirow{2}{*}{ CMS } & PAIN & $2.33 \pm 2.58$ & $1.33 \pm 3.51$ & .887 & .382 \\
\hline & $\mathrm{ADL}$ & $2.80 \pm 2.48$ & $1.60 \pm 2.29$ & 1.375 & .180 \\
\hline
\end{tabular}


Effects of Upper Limb Stabilization Exercise on Pain Control and Improvement of Function in Shoulder

\begin{tabular}{l|c|c|c|c|c|}
\hline & ROM & $4.67 \pm 4.19$ & $2.40 \pm 1.88$ & 1.913 & .066 \\
\cline { 2 - 6 } & POWER & $4.27 \pm 3.90$ & $1.60 \pm 2.47$ & 2.237 & $.033 *$ \\
\cline { 2 - 6 } & Total & $14.80 \pm 12.58$ & $9.00 \pm 11.00$ & 1.344 & .190 \\
\hline
\end{tabular}

\section{Discussions}

The shoulder is a joint that is highly mobile but low in stability, which is used most frequently in a human body. Around $95 \%$ of the patients who experienced dislocation for the first time were due to external forces, such as external injuries or a fall. The dislocation is likely to occur again if it is not properly treated early in the dislocation. The recurrence occurs more often in their twenties, with a probability of up to 95\%. The functional impediment or pain in the shoulder has a significant impact on daily activities and causes a serious problem that degrades one's quality of life.

Bottoni et al. studied a recurrence rate of patients with shoulder dislocation [2]. They found that nine out of 12 patients who had non-surgical treatment $(75 \%)$ and one out of nine patients who had arthroscopy surgery (11.1\%) showed recurrence. However, patients who had surgical treatment had difficulties in daily activities due to the limitation in their joint range of motion after surgery. Thus, their physiotherapy should focus on the recovery of the joint range of motion without pain and prevention or recurrence of shoulder instability by strengthening muscle strength. As found in the report by Bottoni et al., the recurrence rate of patients after surgery was not $0 \%$. Thus, postoperative-management is important [2].

The treatment approach for the shoulder does not separate each of the joints one by one. The movement of the shoulder is complete by the movements of the scapulothoracic joint, glenohumeral joint, acromioclavicular joint, and sternoclavicular joint [12]. Since the shoulder joint is extremely mobile and unstable in the human body, its stability can be raised through muscles. This stabilization is the ability to finely control the joint consciously or unconsciously, and the stabilization of the scapula affects normal movements of the shoulder joint. The scapula is a bone where many muscles are attached to increase the stability of the shoulder joint. Thus, the stabilization of the scapulothoracic joint should be established first, then the glenohumeral joint should stabilize the shoulder joint, although this is not a true joint [13]. The stabilization of the scapula primarily provides stability throughout the shoulder girdle and is also used to correct abnormal movements. The treatment of external rotation of the glenohumeral joint should also be taken in parallel. Since both the abduction and flexion movements of the shoulder require external rotation, pain will occur easily without the correct execution of external rotation movements [14]. Thus, after obtaining the stability of the shoulder girdle, an approach should be taken in the following order: external rotation, flexion, abduction, adduction, extension, and internal rotation in the glenohumeral joint.

Oliveira et al. (2008) reported that the activity of the trapezius increased when shoulder and arm exercises were conducted using the Swiss Ball [8]. Furthermore, Marshall et al. (2005) showed a considerable increase in activity in the rectus abdominis during the core stabilization exercise using the Swiss Ball [4]. They also reported that a long-term stabilization exercise had a significant effect on the functional improvement and reduction in lower back pain [15].

The CMS results in this study were similar to those in the studies by Seo et al. and Jang [16]. However, the Simple Shoulder Test (SST) results in the present study were not statistically significant in the experimental group, which was in contrast with those of Seo et 
al. (2012) [16]. This result was due to the difference in patient groups. The VAS results in the experimental and control groups in this study were similar to the groups in the studies conducted by Seo and Jang [17]. Although the VAS scores did not have statistical significance between groups, the pre-post comparisons within the same groups showed statistical significance in both groups. The experimental group in this study had statistically significant results in active flexion, active abduction, and VAS, and each item and total score of the CMS. The control group in this study had a statistically significant difference in active abduction, SST, and power item in the CMS before and after the treatment. The stabilization exercise had a positive impact on power, functional recovery, and pain control. Active flexion was not statistically significant between groups in the shoulder stabilization movement, but active abduction was statistically significant between groups. Consistency of the shoulder joint is important when the shoulder is abduction, and it can be seen as a benefit to the movement by securing stability.

When comparing the difference before and after the treatment in the experimental and control groups, the joint range of motion, activities of daily living, and total score of the CMS did not show significant values, but the exercise using the Swiss Ball had an effect to some extent, considering the change. This study had limitations in that its experiment period was short and only the pure stabilization exercise was applied and compared within patients who had their shoulder joint capsule repaired.

\section{Conclusions}

This study applied the shoulder stabilization exercise to patients with shoulder dislocation, and evaluated patient pain and shoulder joint functions using the VAS, SST, and CMS. The experimental group had statistically significant results in SST, active abduction, and power item in the CMS. For future studies, the stabilization exercises will be conducted for patients with stroke who had joint capsule repair. The future study will also see which muscles are activated during the exercise using electromyography.

\section{Acknowledgements}

This work was supported by the National Research Foundation of Korea (NRF) grant funded by the Korea government (MSIT) (No-2019R1F1A1057731).

\section{References}

[1] S. Cutts, M. Prempeh, and S. Drew, “Anterior shoulder dislocation,” Annals of the Royal College of Surgeon of England, vol.91, pp.2-7, (2009) DOI:10.1308/003588409X359123

[2] C. R. Bottoni, J. H. Wilckens, T. M. DeBerardino, J. G. D’Alleyrand, R. C. Rooney, J. K. Harpstrite, R. A. and Arciero, "A prospective, randomized evaluation of arthroscopic stabilization versus nonoperative treatment in patients with acute, traumatic, first-time shoulder dislocations," The American Journal of Sports Medicine, vol.30, no.4, pp.576-580, (2002) DOI: 10.1177/03635465020300041801

[3] S. L. Mottram, "Dynamic stability of the Scapula," Manual Therapy, vol.2, no.3, pp.123-131, (1997) DOI: 10.1054/math.1997.0292

[4] H. Osmond-Clarke, "Habitual dislocation of the shoulder," British Orthopedic Association, vol.30B, no.1, pp.19-25, (1948)

[5] K. Hayes, M. Callanan, J. Walton, A. Paxinos, and G. A. C. Murrell, "Shoulder Instability: Management and Rehabilitation," Journal of Orthopedic \& Sports Physical Therapy, vol.32, pp.497-509, (2002) DOI: 10.2519/jospt.2002.32.10.497 
Effects of Upper Limb Stabilization Exercise on Pain Control and Improvement of Function in Shoulder

[6] L. Linsell, J. Dawson, K. Zondervan, P. Rose, T. Randall, R. Fitzpatrick, A. Carr, "Prevalence and incidence of adults consulting for shoulder conditions in UK primary care; patterns of diagnosis and referral," Rheumatology, vol.45, pp.215-221, (2006) DOI: 10.1093/rheumatology/kei139

[7] P. W. Marshall and B. A. Murphy, "Core stability exercises on and off a swiss ball," Archives Physical Medicine and Rehabilitation, vol.86, no.2, pp.242-249, (2005) DOI: 10.1016/J.apmr.2004.05.004

[8] A. S. Oliveira, M. M. Carvalho, and D. P. Cardoso, "Activation of the shoulder and arm muscles during axial load exercises on a stable base of support and on a medicine ball," Journal of Electromyography and Kinesiology, vol.18, no.3, pp.472-479, (2008) DOI: 10.1016/j.jelekin.2006.09.009

[9] B. Stuge, "Evidence of stabilizing exercises for low back- and pelvic girdle pain - a critical review," Brazilian Journal of Physical Therapy, vol.23, no.2, pp.181-186, (2019) DOI: 10.1016/j.bjpt.2018.11.006

[10] S. Green, R. Buchbinder, R. Glazier, and A. Forbes, "Systematic review of randomised controlled trials of interventions for painful shoulder: selection criteria, outcome assessment, and efficacy," BMJ, vol.31, no.316, pp.354-360, (1998) DOI: 10.1136/bmj.316.7128.354

[11] G. Jull, C. Richardson, R. Toppenberg, M. Comerford, and B. Buli, "Towards a measurement of active muscle control for lumbar stabilization,” Australian Journal of Physiotherapy, vol.39, no.3, pp.187-193, (1993) DOI: $10.1016 /$ S0004-9514(14)60481-5

[12] A. J. K. Ostör, C. A. Richards, A. T. Prevost1, C. A. Speed, and B. L. Hazleman, "Diagnosis and relation to general health of shoulder disorders presenting to primary care,” Rheumatology, vol.44, no.6, pp.800-805, (2005) DOI: 10.1093/rheumatology/keh598

[13] M. G. Sayers, S. Lorenzetti, "Influence of technique on upper body force and power production during medicine ball throws," Journal of Sports Sciences, vol.38, no.4, pp.470-475, (2020) DOI: 10.1080/02640414.2019.1706871

[14] C. S. Neer 2nd, "Anterior acromioplasty for the chronic impingement syndrome in the shoulder," The American Orthopedic Association, vol.87, no.6, pp.1399, (1972) DOI: 10.2106/JBJS.8706.cl

[15] K. Yongwook, K. Nayoung, C. Wonhyuk, and L. Sangchul, "Comparison of the therapeutic effects of a sling exercise and a traditional stabilizing exercise for clinical lumbar spinal instability," Journal of Sport Rehabilitation, vol.27, no.1, pp.47-54, (2018) DOI: 10.1123/jsr.2016-0083

[16] S. Hyung-Seok, S. Youn-Bum, L. Jung-Ho, and P. Young-Han, "Effect of extracorporeal shock wave therapy on pain and function in patients with rotator cuff tendinitis," Journal of the Korea Academia-Industrial cooperation Society, vol.13, no.7, pp.3132-3139, (2012)

[17] J. Yeon-woo, B. Sung-soo, and J. Won-sug, "The effect of neural mobilization on the grip strength," Journal of the Korean Society of Physical Medicine, vol.2, no.1, pp.11-20, (2007) 